Modeling

Cooperation in an

Address-register-

based Telephonel

Face-to-face Survey
2016, Vol. 28(4) 396-414

(C) The Author(s) 2016

Reprints and permission:

sagepub.com/journalsPermissions.nav DOI: $10.1177 / 1525822 \times 15617920$

fmx.sagepub.com

@SAGE

\title{
Oliver Lipps'
}

\begin{abstract}
I analyze the effects of household sociodemography, interviewer performance in the current survey, and fieldwork characteristics on cooperation in a central telephone survey, where households with no publicly listed landline number receive face-to-face visits. Using the 2013 refreshment sample of the Swiss Household Panel, I employ household-interviewer cross-classified multilevel models and analyze first and later contacts separately. Some sociodemographic groups are less cooperative in the first contact only, others in both the first and later contacts, and still others in later contacts only. I offer recommendations about which households should be finalized at the first contact, which should be transferred to the face-to-face sample instead of being worked by telephone, and which interviewers should work which household groups.
\end{abstract}

\footnotetext{
' Swiss Centre of Expertise in the Social Sciences (FORS), c/o University of Lausanne, Lausanne, Switzerland
}

\section{Corresponding Author:}

Oliver Lipps, Swiss Centre of Expertise in the Social Sciences (FORS), c/o University of Lausanne, CH - 1015 Lausanne, Switzerland.

Email: oliver.lipps@fors.unil.ch 


\section{Introduction: Adding Face to Face to Telephone Surveys}

Growing telephone undercoverage (Brick et al. 2011; Ernst Staehli 2012; Joye 2012; Joye et al. 2012; Lipps and Kissau 2012; Mohorko et al. 2013) may cause an increasing selection bias in surveys that use the telephone as the sole mode (Busse and Fuchs 2012; Cobben and Bethlehem 2005). Using additional modes for sample members without telephone (e.g., Roberts 2007) may help solve undercoverage issues and improve making contact (Lipps 2016). How to optimize fieldwork to improve cooperation in surveys where an additional mode is used for those without telephone is not clear, however.

The primary aim of this article is to better understand the effects on cooperation from the actors involved in surveys where the primary survey mode is the landline and face to face for households with no telephone. The actors are the households contacted to complete the survey and the interviewers trying to obtain cooperation. To this end, I will use household sociodemography and their fieldwork status and effects from interviewer performance in the current survey. In addition, I analyze if these effects vary by survey mode used. This might shed light on appropriate measures to be taken to eventually increase cooperation.

This article is organized as follows: First, I present an appropriate model of survey cooperation and previous findings of effects from sample members, interviewers, and fieldwork issues. Next, I introduce the data and the modeling approach used and then I discuss the results. I conclude with recommendations to optimize cooperation by (re-)assigning households to the appropriate mode and interviewer and prioritizing contacts.

\section{Actors Involved in Cooperation}

\section{Households}

Generally, to model cooperation of households, their sociodemographic characteristics are used. This is because sociodemography is supposed to influence cooperation and because they are the only variables known of all sample members. Nevertheless, although correlated with cooperation, the causes of (non)cooperation are rather "social participation, ... interest in societal well-being, doing voluntary work, political interest, ... the type of sponsor, and attitudes towards surveys" (Stoop 2005:126). Unfortunately, these variables are not known of unobserved sample members. While household sociodemography is significant for the outcome of the first 
contact with an interviewer, it loses its predictive power of final dispositions for households requiring more than one contact (Groves and Couper 1996). This is because sociodemographic variables with higher cooperation propensities fall out of the sample due to the omission of first-contact respondents. For later contacts, the outcomes of the prior contacts are important indicators for cooperation.

Although sociodemographic variables are generally lacking a causal relationship with survey cooperation, this article will make use of such variables available from the sampling frame where sample members were drawn from. Most of these variables have been shown to correlate with household survey cooperation in both an international (e.g., Groves and Couper 1998; Stoop 2005), and a national context (Lipps 2016; Lipps and Kissau 2012; Lipps et al. 2015). In addition, I will use characteristics of the prior contacts cases of later contacts.

\section{Interviewers}

In the literature, especially in the context of face-to-face surveys, a number of interviewer characteristics were examined to analyze cooperation. Previous research analyzed sociodemographic variables (e.g., Blohm et al. 2007), and most reported no significant interviewer variables (Hox et al. 1991; Pickery et al. 2001). Others checked whether interviewer-sample member matching improves cooperation (Durrant et al. 2010). Still others investigated interviewer experience from earlier surveys (Fowler and Mangione 1990), perceived confidence in their abilities to convince sample members to participate (Durrant et al. 2010; Groves and Couper 1998), and their reported ability to adequately respond to respondent concerns (Morton Williams 1993). Effects on cooperation from these variables are mixed (Blohm et al. 2007).

I was able to test effects on cooperation of most of these variables based on the central telephone part of the survey used in this article. While I found some positive effects of the interviewer confidence in their abilities to convince sample members to participate, this turned out to be insignificant once we control for sample member sociodemography, fieldwork progress, and interviewer performance. It is likely that fixed interviewer characteristics or interviewer-household matching variables lack predictive power in a central telephone context with a random household-interviewer assignment.

As for interviewer experience, it is likely that "most of the acculturation process of producing effective interviewers occurs during training and on 
the job" (Groves and Couper 1998:195; see also Snijkers et al.1999). Groves and Couper's theory of cooperation behavior relies on the characteristics of the interaction between respondent and interviewer (Groves and Couper 1996, 1998; see also Maynard and Schaeffer 1997), rather than on fixed interviewer characteristics. Their concept of "maintaining interaction" (Groves and Couper 1998:chap. 9.3) is based on the strategy of avoiding a termination of the interaction during initial contacts. This concept includes the ability of interviewers of "stepping back" (Hox et al. 1998:174; i.e., accepting a reluctance uttered and returning later as a successful interviewer tactic to react to initially reluctant individuals). It is clear that such tactics can best be learned by training on the job.

Some of the existing models neglect the burden that interviewers face when trying to convince sample members and conduct interviews as an important factor for interviewer performance (Japec 2005). Japec developed a model of interviewer effects on cooperation that acknowledges that interviewer burden - especially the cognitive burden - can have adverse effects. She found evidence that "the number of surveys an interviewer is working on during the same time period, ... the probing frequency, ... the don't know frequency in a survey ... can be stressful" and that "interviewer interest affects the ... refusal rate" (Japec 2006:35). Lipps (2009) went one step further and found adverse effects on cooperation results from negative previous contact performance (the number of refusals obtained). Lipps showed that this was not compensated by positive previous performance (the number of interviews conducted).

To summarize the role of interviewers on cooperation, focusing on effects from prior interviewer performance and experiences collected during the current survey so far seems a promising avenue. These issues were also identified as a research gap in explaining cooperation behavior in the current literature (Durrant et al. 2010).

\section{Household and Interviewer Effects}

In interviewer-based surveys, the variance of the probability to respond has a sample member and an interviewer component. These can be calculated by means of multilevel models with sample members as first and interviewers as second level (e.g., O'Muircheartaigh and Campanelli 1999). Hox et al. (1991) were among the first to calculate the interviewer portion of the variance component $\rho$. They found $\rho=.020$ for interviewers in a mixedmode (telephone and face to face) small controlled field experiment. The interviewer sample was, however, quite homogeneous, received thorough 
training, and used a detailed script to persuade respondents to participate. Pickery et al. (2001) used the second wave of the face-to-face Belgian Election Panel Survey and found $\rho=.044$ (p. 517, Table 3). Japec (2005) reported $\rho=.027$ in the Swedish part of the 2002 face-to-face European Social Survey for the response rate and $\rho=.048$ for the refusal rate. She did not find a positive relationship between interviewer experience and response rates.

\section{Survey Mode}

Most research articles about interviewer effects on cooperation used faceto-face surveys. Although the number of possible stimuli in telephone surveys is smaller than in face-to-face interviews, interviewer effects can still be expected. For example, voice characteristics and speech patterns seem to play a role (Oksenberg and Cannell 1988). In addition, interviewers may not follow directions, have different argumentation skills (de Leeuw and Hox 2004; Snijkers et al. 1999; Stokes and Yeh 1988), or face different kinds and magnitudes of (perceived) burden (Japec 2006). In centralized telephone surveys, several interviewers are typically involved in the completion of sample members who are contacted more than once. So interviewers may contact households that other interviewers have already talked to. This makes both interviewer performance measurement and promising interviewersample member assignments difficult. Generally, interviewer effects measured in centralized telephone surveys tend to be smaller than in faceto-face surveys and higher in first contacts with households than in later contacts (Lipps 2009).

\section{Data}

I use data from the Swiss Household Panel (SHP). The SHP is a yearly conducted, centralized computer-assisted telephone interview (CATI) panel survey representative of the Swiss residential population. Every year, the household reference person is asked to first complete the household grid questionnaire, which includes the household composition and basic sociodemography of the household members. Then, the interview-eligible household members answer the individual questionnaire.

The 2013 refreshment sample (SHP III) was drawn by the Swiss Federal Statistical Office (SFSO) from a frame of individuals residing in Switzerland and based on population registers. The data include a number of sociodemographic variables and a household identifier but no telephone number. 
For surveys that draw their sample from this register but use the telephone as a tool to collect the data, the remaining issue is that the sampling frame itself does not contain telephone numbers. These need to be separately searched and matched to the sample. The SFSO matched the sample against their register of telephone numbers, of which the publicly listed numbers were delivered to the survey agency conducting the SHP survey. I will use sociodemographic variables from the register, telephone-number matching information from the matching procedure, and CATI process ("para") data (Kreuter and Olson 2013). All information is available for both telephone and face-to-face contacts. To summarize, the advantages of using the SHP III sample for the cooperation analysis are as follows:

1. the sample fully covers the target population;

2. sociodemographic information on nonrespondents is only available in this sample;

3. all household members are identifiable for all sample members; and

4. contact data are available from all fielded sample members.

Of the 11,111 households drawn, 7,397 $(66.6 \%)$ could be matched with a publicly listed landline telephone number. A number of studies show that those without a publicly listed landline are generally more difficult to convince to participate (e.g., Joye et al. 2012). All unmatched households are tried to be surveyed using face-to-face interviewers. Altogether, 5,489 households are fielded and successfully contacted by telephone, and 2,267 households are fielded and successfully contacted face to face such that the analysis sample size for modeling cooperation amounts to 7,756 households.

\section{Modeling Cooperation}

We use the final grid completion dummy (Lipps 2008) as dependent variable of a sample household, using all contacts until a final disposition is recorded (American Association for Public Opinion Research [AAPOR] 2015). ${ }^{1}$ This means that all contacts done on a household would be assigned a " 1 " if the household finally participates and " 0 " if not. In the central telephone subsample, where contacts are randomized to interviewers, this measure interprets each contact of the interviewers involved in working the household as one partial contribution to the household cooperation. This dependent variable is in line with Groves and Couper's (1998) "maintaining interaction" model and Hox et al.'s (1998) "stepping back" model, where the ultimate goal of each contact is that the household cooperates. 
With this definition, the telephone and the face-to-face samples can be modeled simultaneously.

Because effects on outcomes of first contacts with households are different than those of later contacts (Groves and Couper 1996; Lipps 2009), I distinguish the models by first and later contacts. Because the telephone and the face-to-face samples are different, I suspect different effects by survey mode and want to learn whether it would be a good idea to contact some person-groups face to face (although they may possibly own a landline), I include a dummy that indicates face-to-face contacted households and interact this dummy with interviewer performance and workload.

I model separately the effects of (1) sample household sociodemography and (2) fieldwork characteristics in terms of number and outcomes of contacts, interviewer performance, and workload.

For (1), I include household sociodemography (age of youngest child in the household and household size; Durrant et al. 2010) and the sociodemography of the household informant, who represents the household as a whole (Durrant and Steele 2009). For the latter, I use the following categories:

- Age-groups: 16-30 years, 31-44 years, 45-58 years, 59-72 years, and $73+$ years.

- Nationality: Swiss or Swiss born, foreigners from one of the neighboring countries, which share one of the Swiss national (survey) languages, foreigners from another country (Font and Mendez 2013).

- Civil status: married, single and never married (hereafter referred to as single), divorced, and widowed.

- Language region: Swiss-German, French, and Italian.

- Size of municipality of residence, representing the degree of urbanization: more than 100,000 inhabitants, 20-100,000 inhabitants, 1020,000 inhabitants, 5-10,000 inhabitants, $2-5,000$ inhabitants, and less than 2,000 inhabitants.

To measure the difficulty to reach the household, we include the number of calls so far (i.e., until the current contact) with no contact attained.

For (2), in terms of short-term interviewer performance, I include the effect of an interviewer's prior contact result (with another household). Contact results are coded as follows:

- Conducting an interview (IW).

- Fixed appointment with the (targeted) household reference person (FA). Typically, a date and time for the interview is fixed in an FA. 
- Vague appointment with the household reference person or another person (VA). A typical VA is household member's response "(You are welcome to) call later" to the interviewer request.

- Soft refusal (including broken appointment; RF).

I expect positive effects from FA or IW (coded as +1 ) and negative effects from RF (coded as -1 ). FA represents a contact result, which should have positive effects in terms of a sense of achievement (the case is ready to be interviewed at the next call). IW represents the workload and interview experience, and RF a negative contact result, for which we would expect frustration effects (Lipps 2009). A VA contact result is coded as 0 . To analyze effects of interviewer performance and interviewer workload as long-term effects, we include the number of the interviewer's contacts so far, distinguished by the number of FAs, IWs, and RFs, respectively.

For later contacts with a household, in addition to the variables used to model first contacts, I include the results of the prior contact (FA and VA). In addition, I distinguish between the regular and the refusal conversion fieldwork phase. While the former includes the contacts until a soft refusals is uttered (if at all), the latter is the fieldwork period during which households that gave a soft refusal are reapproached, after a certain amount of time. I add the number of contacts with the household so far (excluding the number of calls with no contact) to measure the fieldwork effort needed until the current contact. In addition, I include the result of the household's prior contact (FA or VA) to assess the anticipated interviewer difficulty when doing the current contact. I also interact the household's prior contact result (FA or VA) with interviewer experience, with the latter again distinguished by the number of FA, IW, and RF, respectively. The idea is that interviewers with more experience with certain contact results (e.g., "call later," which can be true or a hidden refusal) learn how to better cope with this situation. Finally, I interact the survey mode with the fieldwork variables household number of noncontacts, household number of contacts, and household's prior contact result (FA or VA). To summarize, I list the fieldwork variables in Table 1 .

Regarding the data structure, households are assigned to interviewers at random in the central telephone SHP, thus effectively achieving an interpenetrated design (a random interviewer-respondent assignment; see Mahalanobis 1946). Face-to-face interviewers work households completely. To accommodate the telephone household assignments, I use cross-classified Monte Carlo Markov Chain (MCMC) estimated multilevel models (Fielding and Goldstein 2006; specifically for call data, see Durrant et al. 2010; Durrant 
Table I. Fieldwork Variables Used in Models.

\begin{tabular}{ll}
\hline Variable (As in Modeling Result Table) & Explanation \\
\hline $\begin{array}{l}\text { Interviewer's prior contact (with } \\
\text { another household) FA, IW }=\mathrm{I},\end{array}$ & $\begin{array}{c}\text { Interviewer's contact result with prior } \\
\text { household: fixed appointment (FA) or } \\
\text { interview (IW) }=\mathrm{I}, \text { (soft) refusal }\end{array}$ \\
$\begin{array}{l}\text { Interviewer number of FA } \\
\text { Interviewer number of fixed } \\
\text { appointments so far }\end{array}$ \\
$\begin{array}{l}\text { Interviewer number of IW } \\
\text { Interviewer number of RF } \\
\text { Interviewer number of IW so far } \\
\text { Household prior contact FA }\end{array}$ & $\begin{array}{l}\text { Interviewer number of RF so far } \\
\text { Prior contact on household was FA }\end{array}$ \\
\hline
\end{tabular}

Note: $\mathrm{FA}=$ fixed appointment; $\mathrm{IW}=$ interview; $\mathrm{RF}=$ refusal; $\mathrm{VA}=$ vague appointment.

et al. 2013). Cross-classified means that the data are not hierarchical, but households and interviewers are crossed (via contacts). Bayesian estimation methods such as MCMC consider the model parameters not to have fixed values but uncertain ones. Prior information on this uncertainty is then used along with collected data to update the knowledge of this uncertainty (Fielding and Goldstein 2006). To model the binary dependent variable, I use logistic models and allow for a random interviewer variance.

To estimate the models, I use the MLwiN software (Rasbash et al. 2012), called from within Stata via the runmlwin command (Leckie and Charlton 2013). Starting with a model without covariates to calculate the share of the total variance among the two levels (interviewers and respondents as crossed higher levels), I then add household and household informant sociodemography (including the number of noncontacts) and end up adding interviewer performance and fieldwork characteristics. As a criterion for the model quality, I report the Bayesian deviance information criterion (DIC), which is an MCMC penalized goodness-of-fit measure. It is equivalent to the Akaike information criterion used in maximum likelihood estimation and can be interpreted in a similar way: Given a data set, several competing models may be ranked according to their Bayesian DIC, with the one having the lowest Bayesian DIC being the best. Modeling results with coefficients that are significant on the $1 \%$ level in at least one of the models are depicted in Table 2. The full table can be found in the journal's online section for supplemental materials. 


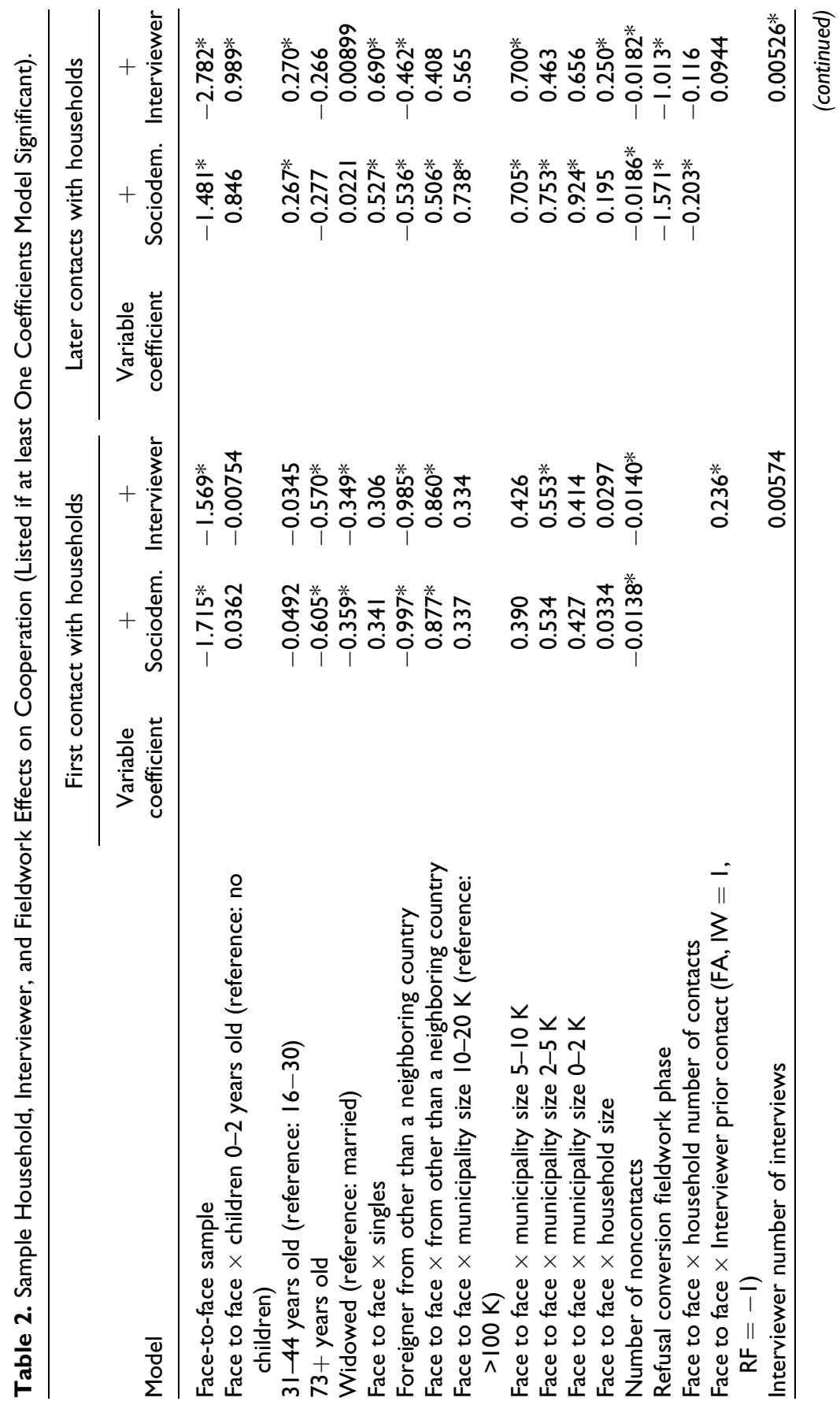




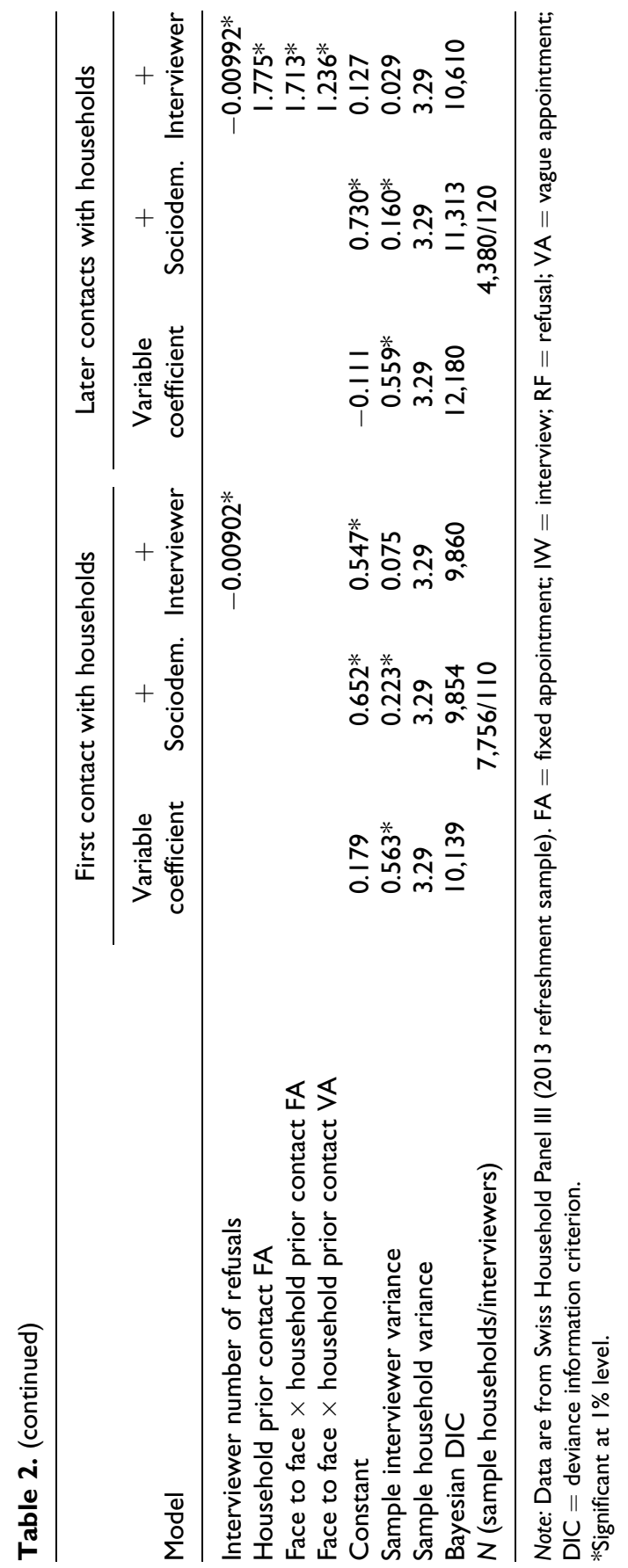




\section{Modeling Results}

\section{First Contact with Households}

Because in binomial models the variance at the lowest level is constraint to the area under the logistic curve $\left(\pi^{2} / 3 \sim 3.29\right.$; see Snijders and Bosker $1999)$, around $15 \%$ of the total variance $(0.563 /[0.563+3.29])$ is due to the interviewers. This figure is considerable and much higher than could be expected from telephone surveys. A large part of the high interviewerspecific variance is due to the fact that interviewers who work the telephone sample and interviewers who work the (more difficult) face-to-face sample are different. This variance is much smaller in the model that includes household sociodemography and is insignificant in the final model, meaning that we can explain the interviewer-specific part of household cooperation almost entirely in the final model.

The second model controls for sample household sociodemography (including sample mode and number of noncontacts). Households that could not be matched with a landline and that are therefore surveyed face to face are more difficult to convince to cooperate. I find less cooperation in older or widowed households. Foreigners with a nationality other than one of the neighboring countries cooperate less than native Swiss or foreigners whose country of birth shares one of the Swiss national languages. This effects is reduced to insignificance in the face-to-face sample. Communication in a less well-mastered language is probably much easier face to face than over the telephone. Finally, the more difficult it is to make contact, the less cooperative is the household.

In the final model, which adds interviewer performance and experience during the survey so far, the Bayesian DIC is slightly larger than in the previous model such that the coefficients need to be carefully interpreted. The variables already included in the previous model change only marginally. I find a positive (short-term) correlation with the result of the interviewer's prior contact in the face-to-face mode. In terms of cumulative effects, only negative experiences play a role: Higher numbers of refusals result in less cooperation at the current contact.

\section{Later Contacts with Households}

When considering later contacts with households, I find a similarly high interviewer variance $(0.559 /[0.559+3.29])$, which again decreases across models and is insignificant in the final model. Note that once more no fixed interviewer characteristics like sociodemography, their confidence to be 
able to persuade reluctant sample members, or interviewer-household matching effects (Durrant et al. 2010) are used to reach an insignificant interviewer variance. This means that almost all interviewer variance can be explained by interviewer and fieldwork variables.

Also similar to first contacts, the face-to-face households are more reluctant than the telephone households. Regarding sociodemography, households with informants between 31 and 44 years of age cooperate more, as did single households when contacted face to face. Also again, foreign households with a nationality other than one of the neighboring countries cooperate less. And again, this effect is reduced to insignificance in the face-to-face sample. Households in smaller municipalities cooperate more in the face-to-face mode. It is interesting that this is not the case at first contacts. Similar to the models using first contacts only, the ones that are more difficult to contact cooperate less, as do households that have uttered a soft refusal (and are reapproached during the refusal conversion phase). A higher number of contacts has adverse effects only when contacted face to face. This may indicate that the "maintaining interaction" tactics does not work well in the (selectively difficult) face-to-face sample.

The final model leaves the variables already in the second model again largely unchanged. There are no short-term interviewer performance effects from prior contacts. As for the long-term effects from their prior contact experiences, I again find adverse effects from cumulated refusals, but also positive effects from the number of successful interviews. Prior contacts with the household resulting in a fixed appointment have positive effects on cooperation, and these effects are stronger in the face-to-face sample. Vague appointments are better than (soft) refusals in the face-to-face mode only. We do not find interaction effects from household prior contact results and interviewer experiences with these results.

\section{Summary and Conclusion}

The aim of this article is to learn more about effects of household sociodemography, interviewer performance and experience collected at the current study so far, and fieldwork status on cooperation in mixed mode telephone/ face-to-face household surveys. To model cooperation, I use data from the 2013 SHP refreshment sample. Households without a matched publicly registered landline received face-to-face visits. Together with sociodemographic information from the population register, I use call ("para") data for both the telephone and the face-to-face contacts. I use multilevel cross-classified logistic models with random interviewer effects and 
contacts at the lowest level. I model first and later contacts with households separately. In both models, there are very high interviewer variances that can be explained almost entirely by household and fieldwork characteristics and interviewer performance and interviewer contact experience made so far.

Some household groups are more or less difficult to convince in both first and later contacts, some in the first, and some in later contacts only. Foreigners from a country that does not share one of the national (survey) languages or are difficult to contact cooperate less in both instances. Households composed of older people are less cooperative in the first contact; households with members aged between 31 and 44 years are more so in later contacts only. There are a few interactions with the survey mode: In the face-to-face mode, singles are easier to convince at later contacts and foreigners from a country without the survey language are easier to convince at first contact. The latter group cooperates no worse when approached face to face. Surveying households living in smaller municipalities have positive cooperation effects in the face-to-face mode only.

Interviewers with a high number of refusals are more likely to produce less cooperative contact results. These effects are true for both first and later contacts. Only in later contacts do interviewers benefit from interview experience in that more interviews are positively correlated with better contact results. In terms of short-term effects, interviewer's results at the prior contact (on another household) are positively correlated with the current contact result at first contacts only, and only in the face-to-face mode. Not surprisingly, prior household contact results are important indicators for the success of the current contact: Fixed appointments at the prior contact are highly probable to be followed by an interview at the current contact. Such positive effects from the prior contact are even stronger in the case of the face-to-face sample. Neutral contact outcomes like vague appointments are better than soft refusals only in the case of the face-to-face mode. Interviewer matching with households in terms of prior contact results according to the interviewer's contact experiences does not pay off.

Based on these findings, I have some recommendations for fieldwork, targeting a high cooperation rate and small sociodemographic cooperation bias:

1. Difficult households at first contact such as hard-to-contact households, with old or widowed members, foreigners who may have problems speaking (one of) the survey language(s), or face-to-face households should be first contacted by the most successful 
interviewers. For foreigners, the hard to contact, and face-to-face households, more than one contact has additional adverse effects on cooperation. These households should be prioritized in the sense that later contacts should be avoided. It could be a good idea to approach foreigners with language problems via the face-to-face mode even if a telephone number was matched.

2. The experience, which interviewers make during the survey, plays a role. Interviewers who have conducted more interviews may increasingly work later contacts, and interviewers who had more refusals should have generally easier households. This seems even more important with increasing fieldwork duration, where an appropriate household-interviewers assignment may pay off in the sense of a responsive fieldwork design (Groves and Heeringa 2006; Wagner 2013).

3. Interviewers should avoid vague appointments ("call later") and especially soft refusals, but instead try to fix a date for an interview, if possible. This is especially the case for the telephone mode.

These recommendations do not take previous bias from noncontact into account (Lipps 2016). Doing so would change the picture somewhat; for example, foreigners are underrepresented in all steps and would need overall prioritization. In contrast, older people are both easier to match and to find and are thus overrepresented in the sample of contacted households. For these people, little cooperation even improves representativeness. In addition, I only consider the household grid fieldwork phase. Subsequent recruitment of individuals within cooperating households can change the picture in addition to the matching and noncontact errors.

A shortcoming of my approach is that while there is a randomized assignment of each telephone contact to interviewers, a face-to-face household is generally worked by one interviewer only. In this design, it is possible to assign interviewers to contacts in the (central) telephone sample according to the household characteristics, prior contact results, and interviewer performance. However, this is logistically much more difficult in the face-to-face sample. Future research may use an experimental householdinterviewer assignment, which would include face-to-face contacts, such as O'Muircheartaigh and Campanelli (1999) did in wave 2 of the British Household Panel Study. Only then could an experimental design be guaranteed, allowing analysis of an optimized assignment of contacts (and interviewers) to households in a mixed-mode telephone/face-to-face survey. 


\section{Acknowledgments}

This study has used the data collected by the Swiss Household Panel (SHP, which is based at the Swiss Centre of Expertise in the Social Sciences (FORS). The project is financed by the Swiss National Science Foundation. The author would like to thank Gian-Andrea Monsch, two anonymous reviewers, and the editor for comments on a previous version of this article.

\section{Declaration of Conflicting Interests}

The author(s) declared no potential conflicts of interest with respect to the research, authorship, and/or publication of this article.

\section{Funding}

The author(s) received no financial support for the research, authorship, and/or publication of this article.

\section{Supplemental Material}

The online data supplements are available at http://fmx.sagepub.com/supplemental.

\section{References}

AAPOR (American Association for Public Opinion Research). 2015. Standard Definitions: Final Dispositions of Case Codes and Outcome Rates for Surveys. 8th ed. AAPOR. http://www.aapor.org/AAPORKentico/AAPOR_Main/media/publications/Standard-Definitions2015_8theditionwithchanges_April2015_logo. pdf (accessed October 26, 2015).

Blohm, M., J. Hox, and A. Koch. 2007. The influence of interviewers' contact behavior on the contact and cooperation rate in face-to-face household surveys. International Journal of Public Opinion Research 19:97-111.

Brick, M., D. Williams, and J. Montaquila. 2011. Address-based sampling for subpopulation surveys. Public Opinion Quarterly 75:409-28.

Busse, B., and M. Fuchs. 2012. The components of landline telephone survey coverage bias. The relative importance of no-phone and mobile-only populations. Quality \& Quantity 46:1209-25.

Cobben, F., and J. Bethlehem. 2005. Adjusting undercoverage and nonresponse bias in telephone surveys. Discussion paper 05006, CBS, Statistics Netherlands, Voorburg/Heerlen, the Netherlands.

de Leeuw, E. D., and J. J. Hox. 2004. I am not selling anything: 29 experiments in telephone introductions. International Journal of Public Opinion Research 16: 464-73. 
Durrant, G. B., J. D’Arrigo, and G. Müller. 2013. Modeling call record data: Examples from cross-sectional and longitudinal surveys. In Improving Surveys with Paradata: Analytic Uses of Process Information, ed. F. Kreuter, 281-308. Hoboken, NJ: John Wiley \& Sons.

Durrant, G. B., R. M. Groves, L. Staetsky, and F. Steele. 2010. Effects of interviewer attitudes and behaviors on refusal in household surveys. Public Opinion Quarterly $74: 1-36$.

Durrant, G. B., and F. Steele. 2009. Multilevel modelling of refusal and noncontact nonresponse in household surveys: Evidence from six UK government surveys. Journal of the Royal Statistical Society, Series A 172:361-81.

Ernst Staehli, M. 2012. Telephone surveys in Switzerland: Spotlight. In Telephone Surveys in Europe: Research and Practice, eds. M. Häder, S. Häder, and M. Kühne, 25-36. Heidelberg, Germany: Springer.

Fielding, A., and H. Goldstein. 2006. Cross-classified and multiple membership structures in multilevel models: An introduction and review. Research Report RR791, Department for Education and Skills, University of Birmingham, Birmingham, UK.

Font, J., and M. Méndez, eds. 2013. Surveying Ethnic Minorities and Immigrant Populations: Methodological Challenges and Research Strategies. Amsterdam: Amsterdam University Press.

Fowler, F. J., and T. Mangione. 1990. Standardized Survey Interviewing: Minimizing Interviewer-related Error. Newbury Park, CA: Sage.

Groves, R., and M. Couper. 1996. Contact-level influences on cooperation in faceto-face surveys. Journal of Official Statistics 12:63-83.

Groves, R., and M. Couper. 1998. Nonresponse in Household Interview Surveys. New York: Wiley.

Groves, R., and S. Heeringa. 2006. Responsive design for household surveys: Tools for actively controlling survey errors and costs. Journal of the Royal Statistical Society, Series A 169:439-58.

Hox, J., E. de Leeuw, and I. Kreft. 1991. The effect of interviewer and respondent characteristics on the quality of survey data: A multilevel model. In Measurement Errors in Surveys, eds. P. Biemer, R. Groves, L. Lyberg, N. Mathiowetz, and S. Sudman, 439-61. New York: Wiley.

Hox, J., E. de Leeuw, and G. Snijkers. 1998. Fighting nonresponse in telephone interviews; Successful interviewer tactics. ZUMA Nachrichten Spezial 4: $173-85$.

Japec, L. 2005. Quality issues in interviewer surveys: Some contributions. Ph.D. thesis, Department of Statistics, Stockholm University, Stockholm.

Japec, L. 2006. Quality issues in interview surveys-Some contributions. Bulletin de Méthodologie Sociologique 90:26-42. 
Joye, C. 2012. SRPH-castem. FORS-SFSO workshop, Neuchâtel, Switzerland, June 21.

Joye, D., A. Pollien, M. Sapin, and M. Stähli. 2012. Who can be contacted by phone? Lessons from Switzerland. In Telephone Surveys in Europe, eds. M. Häder, S. Häder, and M. Kühne, 85-102. Heidelberg, Germany: Springer.

Kreuter, F., and K. Olson. 2013. Paradata for nonresponse error investigation. Improving surveys with paradata. In Improving Surveys with Paradata: Analytic Uses of Process Information, ed. F. Kreuter, 13-42. Hoboken, NJ: John Wiley \& Sons.

Leckie, G., and C. Charlton. 2013. runmlwin-A program to run the MLwiN multilevel modelling software from within Stata. Journal of Statistical Software $52: 1-4$.

Lipps, O. 2008. A note on interviewer performance measures in centralised CATI surveys. Survey Research Methods 2:61-73.

Lipps, O. 2009. Cooperation in centralised CATI household panel surveys-A contact-based multilevel analysis to examine interviewer, respondent, and fieldwork process effects. Journal of Official Statistics 25:323-38.

Lipps, O. 2016. Non-observation bias in an address-register-based CATI/CAPI mixed mode survey. Methods, Data, Analysis 1.

Lipps, O., and K. Kissau. 2012. Nonresponse in an individual register sample telephone survey in Lucerne (Switzerland). In Telephone surveys in Europe, eds. M. Häder, S. Häder, and M. Kühne, 187-208. Heidelberg, Germany: Springer.

Lipps, O., N. Pekari, and C. Roberts. 2015. Undercoverage and nonresponse in a listsampled telephone election survey. Survey Research Methods 2:71-82.

Mahalanobis, P. 1946. Recent experiments in statistical sampling in the Indian Statistical Institute. Journal of the Royal Statistical Society 109:325-70.

Maynard, D. W., and N. C. Schaeffer. 1997. Keeping the gate: Declinations to the request to participate in a telephone survey interview. Sociological Methods and Research 26:34-79.

Mohorko, A., E. de Leeuw, and J. Hox. 2013. Coverage bias in European telephone surveys: Developments of landline and mobile phone coverage across countries and over time. Survey Methods: Insights from the Field. http://surveyinsights. org $/ \mathrm{p}=828$ (accessed January 5, 2016).

Morton Williams, J. 1993. Interviewer Approaches. Aldershot, UK: Dartmouth.

Oksenberg, L., and C. Cannell. 1988. Effects of interviewer vocal characteristics on nonresponse. In Telephone Survey Methodology, eds. R. Groves, P. Biemer, L. Lyberg, J. Massey, W. Nicholls II, and J. Waksberg, 257-72. New York: Wiley. O'Muircheartaigh, C., and P. Campanelli. 1999. A multilevel exploration of the role of interviewers in survey non-response. Journal of the Royal Statistical Society, Series A 162:437-46. 
Pickery, J., G. Loosveldt, and A. Carton. 2001. The effects of interviewer and respondent characteristics on response behaviour in panel surveys. Sociological Methods \& Research 29:509-23.

Rasbash, J., F. Steele, W. Browne, and H. Goldstein. 2012. A User's Guide to MLwiN, v2.26. Bristol, UK: Centre for Multilevel Modelling, University of Bristol.

Roberts, C. 2007. Mixing modes of data collection in surveys: A methodological review. National Centre for Research Methods, Review Papers NCRM/008, London.

Snijders, T., and R. Bosker. 1999. Multilevel analysis. Newbury Park, CA: Sage.

Snijkers, G., J. Hox, and E. de Leeuw. 1999. Interviewer's tactics for fighting survey nonresponse. Journal of Official Statistics 15:185-98.

Stokes, L., and M.-Y. Yeh. 1988. Searching for causes of interviewer effects in telephone surveys. In Telephone Survey Methodology, eds. R. Groves, P. Biemer, L. Lyberg, J. Massey, W. Nicholls II, and J. Waksberg, 357-76. New York: Wiley. Stoop, I. 2005. The hunt for the last respondent: Nonresponse in sample surveys. Ph.D. thesis, Utrecht University, Utrecht, the Netherlands.

Wagner, J. 2013. Adaptive contact strategies in telephone and face-to-face surveys. Survey Research Methods 7:45-55. 